\title{
ON PRÜFER RINGS AS IMAGES OF PRÜFER DOMAINS
}

\author{
MONTE B. BOISEN, JR. AND MAX D. LARSEN
}

\begin{abstract}
Only commutative rings with unity will be considered in this paper. It is shown that if $R$ is the homomorphic image of a Prüfer domain, then $R$ is a Prüfer ring but that the converse is not true in general. It is then shown that a Prüfer ring $R$ is the homomorphic image of a Prüfer domain if and only if the total quotient ring of $R$ is the homomorphic image of a Prüfer domain. A class of total quotient rings which satisfy this last condition is then presented.
\end{abstract}

Only commutative rings with unity will be considered in this paper. An element of a ring is said to be a regular element in case it is not a zero divisor and an ideal is said to be regular in case it contains a regular element. Griffin [2] defined a ring to be Prüfer if every finitely generated regular ideal is invertible. Griffin shows that $R$ is a Prüfer ring if and only if $A(B \cap C)=A B \cap A C$ for all ideals $A, B, C$ of $R$, with $B$ or $C$ regular. Prüfer rings have been studied in [1], [2], [3], and an account of Prüfer rings can be found in [4].

First we will give a necessary condition for a ring to be a homomorphic image of a Prüfer domain. Then we will give an example of a Prüfer ring which does not satisfy the condition, thus providing a negative answer to a question asked informally by Robert Gilmer, namely that Prüfer rings are not necessarily images of Prüfer domains.

THEOREM 1. If $R$ is a homomorphic image of a Prüfer domain $D$, then $A(B \cap C)=A B \cap A C$ for any ideals $A, B$, and $C$ of $R$. In particular, $R$ is a Prüfer ring.

Proof. We always have $A(B \cap C) \subseteq A B \cap A C$. We may assume that $A$, $B$, and $C$ are nonzero, for the contrary case presents no problem. Let $\phi$ be the homomorphism from $D$ onto $R$ and let $A^{\prime}, B^{\prime}, C^{\prime}$ be the preimages under $\phi$ of $A, B$, and $C$ respectively. Let $x \in A B \cap A C$, say $x=\sum a_{i} b_{i}=$ $\sum d_{j} c_{j}$ for $a_{i}, d_{j} \in A, b_{i} \in B, c_{j} \in C$. Let $a_{i}^{\prime}, d_{j}^{\prime} \in A^{\prime}, b_{i}^{\prime} \in B^{\prime}$, and $c_{j}^{\prime} \in C^{\prime}$ be any preimages of $a_{i}, d_{j}, b_{i}, c_{j}$ respectively. Let $x^{\prime}=\sum a_{i}^{\prime} b_{i}^{\prime}$. Then $\phi\left(x^{\prime}\right)=x$ and since $\phi\left(\sum d_{j}^{\prime} c_{j}^{\prime}\right)=x$, we have $x^{\prime}=\sum a_{i}^{\prime} b_{i}^{\prime}=\sum d_{j}^{\prime} c_{j}^{\prime}+k^{\prime}$ where $k^{\prime} \in K^{\prime}$, the kernel of $\phi$. Hence $x^{\prime} \in A^{\prime} B^{\prime} \cap\left(A^{\prime} C^{\prime}+K^{\prime}\right)$. But since $D$ is a Prüfer domain,

AMS (MOS) subject classifications (1970). Primary 13F05; Secondary $13 B 99$. 
$A^{\prime} B^{\prime} \cap\left(A^{\prime} C^{\prime}+K^{\prime}\right)=\left(A^{\prime} B^{\prime} \cap A^{\prime} C^{\prime}\right)+\left(A^{\prime} B^{\prime} \cap K^{\prime}\right)=A^{\prime}\left(B^{\prime} \cap C^{\prime}\right)+\left(A^{\prime} B^{\prime} \cap K^{\prime}\right)$. Hence $x=\phi\left(x^{\prime}\right) \in A(B \cap C)$. Consequently $A(B \cap C)=A B \cap A C$.

Now, for the example, let $F$ be a field of characteristic not equal to two and let $X$ and $Y$ be indeterminates over $F$. Let $R=F[X, Y] /\left(X^{2}, Y^{2}\right)$ and make the obvious identification of images. We note that $a+b X+c Y+$ $d X Y$ is regular if and only if $a \neq 0$ and in this case,

$$
a^{-1}+\left(-b a^{-2}\right) X+\left(-c a^{-2}\right) Y+\left(2 c b a^{-3}-d a^{-2}\right) X Y
$$

is its inverse. Thus $R$ is its own total quotient ring and therefore a Prüfer ring. Let $A=X R, B=(X-Y) R$ and $C=(X+Y) R$. Clearly $A B \cap A C=$ $(X Y) R$. If $z \in B \cap C$, then

$$
\begin{aligned}
z & =\left(a_{1}+b_{1} X+c_{1} Y+d_{1} X Y\right)(X-Y) \\
& =\left(a_{2}+b_{2} X+c_{2} Y+d_{2} X Y\right)(X+Y)
\end{aligned}
$$

where $a_{1}, a_{2}, b_{1}, b_{2}, c_{1}, c_{2}, d_{1}, d_{2} \in F$. Hence

$$
a_{1} X-a_{1} Y+c_{1} X Y-b_{1} X Y=a_{2} X+a_{2} Y+c_{2} X Y+b_{2} X Y \text {. }
$$

Therefore $a_{1}=a_{2}$ and $a_{1}=-a_{2}$ which implies that $a_{1}=a_{2}=0$ since $F$ does not have characteristic two. Consequently, $z \in X Y R$ and $A(B \cap C)=$ $A((X Y) R)=(0)$ which shows that $A B \cap A C \neq A(B \cap C)$. By Theorem $1, R$ is a Prüfer ring which is not a homomorphic image of a Prüfer domain.

Now that we have established the fact that not all Prüfer rings can be realized as homomorphic images of Prüfer domains, we turn to the natural question of which ones can be so realized. While this question will not be completely answered here, we will show that it is sufficient to settle the question for total quotient rings. If $A$ is an ideal of $R$, denote the fractional ideal $R: A$ by $A^{-1}$. Then $A$ is invertible if and only if $A A^{-1}=R$.

THEOREM 2. A Prüfer ring is the homomorphic image of a Prüfer domain if and only if its total quotient ring is the homomorphic image of a Prüfer domain.

Proof. Let $R$ be a Prüfer ring with total quotient ring $T$. Suppose that $R$ is the homomorphic image of the Prüfer domain $D$ under the homomorphism $\theta$. Then let $S=\{s \in D \mid \theta(s)$ is a regular element of $R\}$ and let $\phi$ be the extension of $\theta$ to $D_{S}$ obtained by $\phi(d / s)=\theta(d) / \theta(s)$. Then it is easy to see that $\phi$ is a homomorphism from the Prüfer domain $D_{S}$ onto $T$.

Now let us assume that $T$ is the homomorphic image of the Prüfer domain $K$ under the homomorphism $\phi$, say. Let $U=\{k \in K \mid \phi(k)$ is a unit in $T\}$. Let $\psi$ be defined from $K_{U}$ to $T$ by $\psi(k / u)=\phi(k) \phi(u)^{-1}$. One can easily show that $\psi$ is a homomorphism and $K_{U}$ is clearly a Prüfer domain. Hence, without loss of generality, we may assume that $\phi(k)$ is a unit in $T$ if and only if $k$ is a unit in $K$. Let $D=\{k \in K \mid \phi(k) \in R\}$ and let $\theta$ be the 
restriction of the mapping $\phi$ to $D$. Let $S=\{d \in D \mid \phi(d)$ is a regular element of $R$ \}. Since $\phi(s)$ is a unit in $T, s^{-1} \in K$ and hence $D_{S} \subseteq K$. Let $k \in K$. Then $\phi(k)=\theta(d) / \theta(s)$ for some $d \in D, s \in S$. Therefore, $k=(d / s)-t$ where $t$ belongs to the kernel of $\phi$ which is the kernel of $\theta$, and thus $t$ is in $D$. Hence, $k=(d-t s) / s \in D_{S}$. Therefore $K=D_{S}$.

Let $A=\left(a_{1}, a_{2}, \cdots, a_{n}\right)$ be an ideal of $D$ such that $\theta(A)$ is a regular ideal of $R$. Then there exist elements $t_{1}, t_{2}, \cdots, t_{n} \in \theta(A)^{-1}$ such that $\sum_{i=1}^{n} t_{i} \theta\left(a_{i}\right)=1$. Let $e_{i} \in K$ be such that $\phi\left(e_{i}\right)=t_{i}, i=1, \cdots, n$. Then $e_{i} \in$ $A^{-1}$ for all $i$ since $\phi\left(e_{i} a_{j}\right)=\phi\left(e_{i}\right) \phi\left(a_{j}\right)=t_{i} \theta\left(a_{j}\right) \in R$ and hence $e_{i} a_{j} \in D$ which implies that $e_{i} A \subseteq D$ and consequently $e_{i} \in A^{-1}$. Since $\phi\left(\sum e_{i} a_{i}\right)=1$ is a unit in $R, \sum e_{i} a_{i}$ is a unit in $K$ and its inverse is an element of $D$. Therefore, $\sum e_{i} a_{i}$ is a unit in $D$ and hence $A A^{-1}$ contains a unit which implies that $A$ is invertible. Now let $a, b \in D$ such that $\theta(a)$ and $\theta(b)$ are zero divisors. Since $a$ and $b$ generate an invertible ideal in $K$, there exist elements $c^{\prime}, d^{\prime} \in$ $((a, b) K)^{-1}$ such that $a c^{\prime}+b d^{\prime}=1$. Since $a c^{\prime}, a d^{\prime}, b c^{\prime}, b d^{\prime} \in K$, there exists an element $s \in S$ such that $s c^{\prime}, s d^{\prime} \in((a, b) D)^{-1}$, and if $s c^{\prime}=c$ and $s d^{\prime}=d$, then $a c+b d=s$. Therefore $B=(a, b, a c, a d, b c, b d)$ is a finitely generated ideal of $D$ such that $\theta(B)$ is a regular ideal of $R$. Hence, by the case considered above, $B$ is invertible and so there exist elements $e, f, g, h, i, j \in B^{-1}$ such that

$$
a e+b f+a c g+a d h+b c i+b d j=1 \text {. }
$$

But clearly $e, f, c g, d h, c i, d j \in((a, b) D)^{-1}$ and hence $(a, b) D$ is an invertible ideal of $D$. Therefore $D$ is a Prüfer domain.

We are now in a position to describe a class of Prüfer rings which are homomorphic images of Prüfer domains. Let $L$ be a field of characteristic $n$ and let $K$ be the direct sum of $m$ copies of $L$ where $m \leqq n$ if $n \neq 0$ and $m$ is unrestricted if $n=0$. With this notation in hand we can prove the following.

THEOREM 3. Let $R$ be a Prüfer ring with total quotient ring $K$ where $K$ is as defined in the above paragraph. Then $R$ is the homomorphic image of a Prüfer domain.

Proof. Let $M_{i}=(X-i) L[X]_{(X-i)}, i=1, \cdots, m$. (Here $i$ is the identity of $L$ added to itself $i$ times.) Let $V_{i}=L+M_{i}$ for each $i=1, \cdots, m$. Let $f(X) / g(X)$ be an element of the quotient field of $V_{i}$. Then we can write $f(X) / g(X)=\left(f^{\prime}(X) / g^{\prime}(X)\right)(X-i)^{t}$ where neither $f^{\prime}(X)$ nor $g^{\prime}(X)$ is divisible by $(X-i)$. If $t \geqq 0, f(X) / g(X) \in V_{i}$ and if $t<0, g(X) / f(X) \in V_{i}$; hence $V_{i}$ is a valuation ring with maximal ideal $M_{i}$. Therefore, $V=\bigcap_{i=1}^{m} V_{i}$ is a Prüfer domain with maximal ideals $M_{1} V, \cdots, M_{m} V$. Let $N=\bigcap_{i=1}^{m} M_{i} V$ and set $\bar{V}=V / N$. Now, in $\bar{V}$, the ideals $\overline{M_{1} V}, \cdots, \overline{M_{m} V}$ form a pairwise comaximal set. Hence $\bar{V}=R_{1} \oplus R_{2} \oplus \cdots \oplus R_{m}$ where $R_{i} \cong \bar{V} \mid \overline{M_{i} V}, i=1, \cdots, m$. But $\bar{V}\left|\overline{M_{i} V} \cong V\right| M_{i} V$ and we note that $M_{i} V$ is precisely the kernel of the 
homomorphism from $V$ to $L$ defined by replacing the indeterminate $X$ with $i$. Hence $R_{i} \cong V / M_{i} V \cong L$. Therefore $K \cong R_{1} \oplus \cdots \oplus R_{m}$ and so $K$ is the homomorphic image of the Prüfer domain $V$.

\section{REFERENCES}

1. Monte B. Boisen, Jr. and Max D. Larsen, Prüfer and valuation rings with zero divisors, Pacific J. Math. 40 (1972), 7-12.

2. Malcolm Griffin, Prüfer rings with zero divisors, J. Reine Angew. Math. 239/240 (1969), 55-67. MR 41 \#188.

3. Max D. Larsen, Prüfer rings of finite character, J. Reine Angew. Math. 247 (1971), 92-96. MR 43 \#3255.

4. Max D. Larsen, and Paul J. McCarthy, Multiplicative theory of ideals, Academic Press, New York, 1971.

Department of Mathematics, Virginia Polytechnic Institute and State UniVERSITY, BLACKSBURG, VIRGINIA 24061

Department of Mathematics, University of Nebraska, Lincoln, Nebraska 68508 